\title{
Apropiación territorial e informalidad urbana. Un caso en el Partido de La Plata, Buenos Aires, Argentina
}

\section{Territorial appropriation and urban informality A case in La Plata, Buenos Aires, Argentina}

\author{
Rocío Rodríguez Tarducci \\ rociotarducci@gmail.com \\ Instituto de Investigaciones y Políticas del Ambiente \\ Construido (IIPAC) - Facultad de Arquitectura y \\ Urbanismo (FAU), Universidad Nacional de La Plata \\ (UNLP) - CONICET, Argentina

\section{Daniela Cortizo \\ Instituto de Investigaciones y Políticas del Ambiente Construido (IIPAC) - Facultad de Arquitectura y (UNLP) - CONICET, Argentina} \\ Urbanismo (FAU), Universidad Nacional de La Plata \\ Julieta Frediani \\ Instituto de Investigaciones y Políticas del Ambiente \\ Construido (IIPAC) - Facultad de Arquitectura y \\ Urbanismo (FAU), Universidad Nacional de La Plata \\ (UNLP) - CONICET, Argentina
}

Recepción: 03 Junio 2020

Aprobación: 12 Agosto 2020

Publicación: 02 Noviembre 2020

Cita sugerida: Rodríguez Tarducci, T., Cortizo, D. y Frediani, J. (2020). Apropiación territorial e informalidad urbana. Un caso en el Partido de La Plata, Buenos Aires, Argentina. Geograficando, 16(2), e077. https://doi.org/10.24215/2346898Xe077
Resumen: El presente artículo propone el análisis de la apropiación territorial en el barrio Puente de Fierro, una urbanización informal en la periferia urbana del Partido de La Plata, Buenos Aires, Argentina. La investigación se desarrolla metodológicamente a partir del trabajo de campo y la participación activa y colaborativa con los actores sociales al interior del caso de estudio. La experiencia en al barrio y con los actores contribuyó a la comprensión del valor simbólico que se le otorga a un territorio con características de lugar. De esta investigación surge que los habitantes del caso de estudio se apropian del territorio al nombrar su barrio, recorrerlo, usarlo y representarlo tanto simbólica como materialmente. El análisis permite conocer los lugares en donde se establecen las relaciones sociales y se fortalece el vínculo con el territorio, es decir, mediante la territorialización del significado delas representaciones sociales de los actores.

Palabras clave: Urbanizaciones informales, Apropiación, Metodología, Caso de estudio.

Abstract: This article proposes the analysis of territorial appropriation in the Puente de Fierro neighborhood, an informal urbanization in the urban periphery of the La Plata, Buenos Aires, Argentina. Research is developed methodologically from fieldwork as well as active and collaborative participation with social actors within the case of study. The experience in the neighborhood and with the actors contributed to understanding the symbolic value that is given to a territory with local characteristics. From this research, it emerges that inhabitants of the case study appropriate the territory by naming their neighborhood, touring it, using it and representing it, both symbolically and materially. The analysis makes it possible to know the places where social relations are established and the link with the territory is strengthened; that is, by territorializing the meaning of the actors' social representations.

Keywords: Informal urbanizations, Appropriation, Methodology, Study case. 


\section{A) INTRoducción}

La informalidad urbana-entendida como la cualidad que presenta todo tipo de asentamiento cuyas formas de ocupación del suelo no se ajustan a las normas urbanísticamente establecidas ni a parámetros ambientales, y en los que las características físicas del entorno revisten cierto grado de precariedad-se ha convertido durante las últimas décadas en una problemática que afecta a una gran cantidad de habitantes. A nivel mundial puede estimarse que alrededor de un cuarto de la población urbana vive en barrios en condiciones de informalidad, sin infraestructura de servicios urbanos básicos y sin seguridad de tenencia del suelo (ONU-hábitat, 2015). En este sentido, las ciudades de América Latina se encuentran entre las más desiguales del mundo. Allí este tipo de urbanizaciones se han convertido en la principal estrategia de acceso al suelo para los sectores de menores recursos económicos de la población. El presente trabajo propone el análisis de la apropiación territorial y del valor simbólico que se le atribuye a un lugar. Dicho valor es entendido como una categoría a partir de la cual es posible analizar las relaciones complejas y variadas que se establecen entre los actores y el territorio, al transformarlo y dotarlo de sentido, y con el que se establecen relaciones identitarias. Se toma como caso de estudio uno del interior de una de las 164 urbanizaciones informales del Partido de La Plata, que corresponde con el barrio Puente de Fierro ${ }^{1}$ (Figura 1). Este barrio se considera una de las urbanizaciones informales más grandes del Partido de La Plata y con mayor número de habitantes (alcanza los 5.200) (Rodríguez Tarducci, 2020).

Figura 1. Localización del Barrio Puente de Fierro.

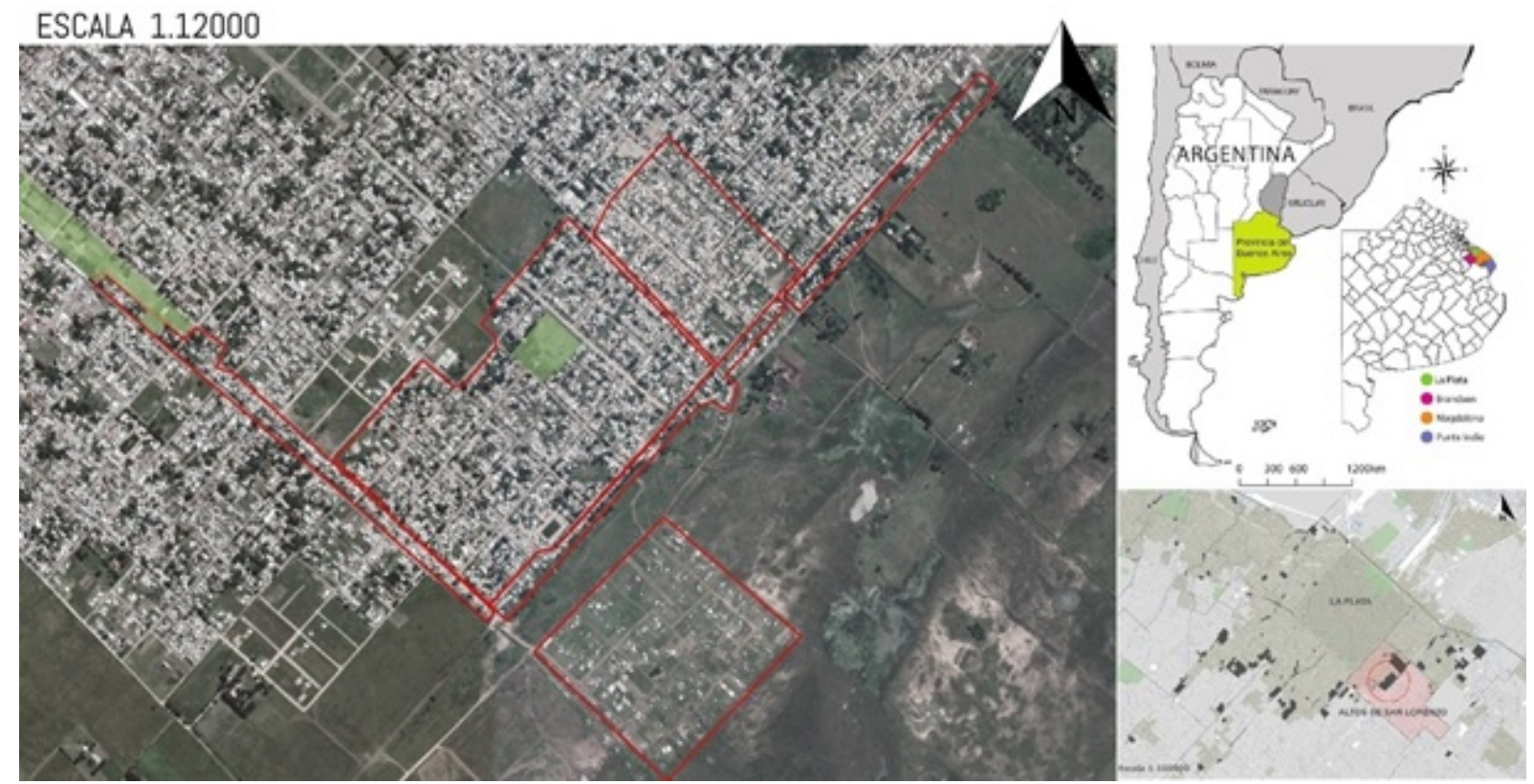

Fuente. Elaboración propia, 2020.

Para el análisis se pone especial atención en las prácticas de los habitantes a la luz de los contextos político-sociales y en las representaciones de los sentidos que los propios actores tienen sobre el territorio. Se incluye la dimensión simbólica, ya que, como afirma Reguillo (1996), en ocasiones se confunde el "escenario situacional (la marginación, la pobreza, la exclusión)" con las representaciones internas de los habitantes de las urbanizaciones informales, o se establece simplemente una relación mecánica entre las prácticas y sus representaciones. 


\section{B) Marco té́rico}

En primer lugar, y para una mejor comprensión de la problemática, previo al análisis de la apropiación territorial aplicada al caso de estudio, resulta necesario definir brevemente aquellos conceptos que guían la investigación, en los cuales se fundamenta y sostiene la postura teórica y epistemológica a lo largo del presente artículo. En ese sentido, los principales conceptos que se utilizan en este trabajo parten del entendimiento dela complejidad que implican ciertos procesos socio territoriales centrales en este trabajo, tales como la apropiación y la informalidad urbana. En este sentido, el primer concepto que se presenta, a modo de macro concepto, es el de territorio, entendido el mismo como una construcción social, en la que toda relación entre actores tiene ocurrencia y se expresa como territorialidad, y en la que se destaca la importancia que tiene el actor en la definición del mismo. El concepto de territorio ha sido históricamente entendido como el escenario de las relaciones sociales, pero las actividades de los actores o grupos sociales varían contextualmente, y por lo tanto la capacidad real y potencial de crear, recrear y apropiar es desigual según cada actor o grupo social, y por este motivo cada territorio merece un análisis particular de acuerdo a su complejidad.

En cuanto al concepto de lugar, que también resulta clave para el análisis de la apropiación, autores como Bourdieu (2000) lo utilizan para referirse a espacios de lucha entre actores sociales con intereses diversos. A pesar de que el concepto de lugar en principio parece aludir a un espacio con límites definidos, dichos límites se extienden hasta donde lo hace el contenido simbólico de los elementos objetivados en él, que pueden ampliarse a través de redes sociales y relaciones de poder. Por este motivo también se suele considerar al lugar como una acumulación de significados (Gumuchian, 1991).

Por último, el concepto de apropiación se convierte en el concepto hacia el que convergen los demás y donde cobran sentido para este trabajo, ya que, según lo expuesto, la apropiación se produce a partir del vínculo entre los actores y los territorios, simultáneamente con la creación de un lugar. En este sentido, aquellos territorios apropiados serían los utilizados para cubrir las necesidades y las posibilidades de un grupo determinado, y se transforman en lugares de apropiación simbólica e identidad (Schneider; Tartaruga, 2006). Uno de los referentes teóricos en relación al concepto de apropiación del año 2005 es el trabajo llevado a cabo por Tomeu Vidal Moranta y Enric Pol Urrútia, cuyo interés parte de disciplinas como la psicología social y ambiental. En este sentido, los autores plantean una propuesta teórica que sirva para comprender la vinculación entre las personas y los lugares. Resulta interesante este abordaje del concepto de apropiación, basado en los vínculos que se establecen entre las personas y el territorio, si entendemos que los lugares se construyen socialmente, y que de esa interacción pueden surgir algunas categorías de análisis tales como el valor simbólico, la identidad y el apego al lugar. La línea de trabajo llevada adelante por Vidal y Pol se interesa en las formas de construir las relaciones de los actores con el territorio. En este sentido, para estos autores, y de igual modo para este trabajo, el énfasis está puesto en analizar la relación entre las experiencias cotidianas y los sentidos de lugar que construyen los actores, con énfasis en las acciones que se llevan a cabo en un territorio y tiempo histórico determinado.

\section{c) Metodología}

En cuando a la metodología adoptada para llevar a cabo el análisis de la apropiación territorial, en primer lugar, se parte del establecimiento de una relación conceptual entre la apropiación y la creación de sentido de lugar, entendida como el resultado entre las acciones y los atributos físicos del espacio. Entre las herramientas que se utilizaron para la recolección de información se encuentran entrevistas realizadas a los principales referentes barriales que formaron parte del trabajo de campo durante la realización de la tesis de doctorado en geografía de quien escribe, denominada Informalidad Urbana en el Partido de La Plata: Análisis del proceso de ocupación y apropiación territorial, 1989 - Actualidad (2020),y el censo puerta a puerta que se llevó a cabo en 
el año 2017 en Puente de Fierro, denominado “Trabajo digno, identidades, necesidades y sueños”, en el marco del Proyecto de Investigación Orientado (PIO) UNLP CONICET “Gestión Integral del Territorio", 2 en el que se realizaron algunas preguntas a los vecinos acerca de los cambios que podían observar en el barrio y su opinión acerca de ellos. Del mismo modo se recurrió, como herramienta de recolección de datos, a la observación directa y a la participación en actividades con los referentes, entre las que se destaca la participación en las "mesas de trabajo permanente"3 (en adelante MTP). Se tiene en cuenta para el análisis que la idea de apropiación remite a la transformación de un espacio con características intrínsecas en un lugar significativo desde la experiencia del actor. Es posible, entonces, identificar un lugar a través a través del establecimiento de dimensiones que operacionalizan el análisis de la apropiación territorial, como las acciones, la identificación simbólica y el apego al lugar, tal como se observa en la figura 2.

Figura 2. Operacionalización del concepto de apropiación.

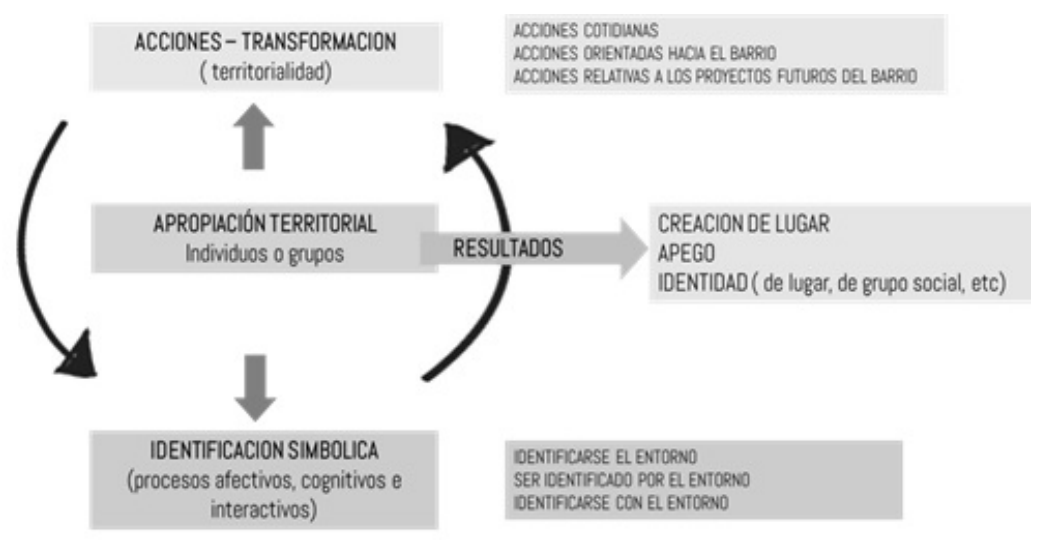

Fuente. Elaboración propia, a partir de Vidal y Pol, 2005.

Los objetos en el territorio se convierten en testimonios de las acciones, su contenido es estructurado a través de un devenir espacio-temporal de acciones, que los provee de funciones diversas en cada etapa del proceso de totalización. En este contexto, las acciones, en tanto proceso, se vinculan directamente con la idea de praxis, esto es, las prácticas sociales que participan de la producción de un orden, cuya naturaleza es, simultáneamente, jerárquica y cotidiana (Santos, 1996a). Son varias las posturas teóricas, las dimensiones y los elementos que componen el proceso de la apropiación como tal, pero en este trabajo se retoma la propuesta de Vidal y Pol (2005), debido a que la misma no describe al territorio solamente como una referencia geográfica en la cual se suscitan las prácticas sociales, sino que, por el contrario, afirma que los grupos o individuos, al ocupar y apropiarse de un territorio, lo transforman físicamente (acción-transformación), dotándolo a su vez de características simbólicas que lo posicionan como un lugar (identificación simbólica). El concepto de apropiación, entonces, desde esta perspectiva puede ser explicado a través de la identificación, la acción en el entorno (el barrio en este caso), y el apego al mismo. Para dar cuenta de ello se establecen tres dimensiones de análisis: la acción, la identificación y el apego al barrio como se indica en la figura 3. 
Figura 3. Dimensiones, componentes e indicadores de la apropiación territorial.

\begin{tabular}{|c|c|c|}
\hline DIMENSION & OMFONENIE & INDCADOFES \\
\hline $\begin{array}{l}\text { AFEGOAL } \\
\text { WGAR }\end{array}$ & SМBáனCA & $\begin{array}{l}\text { Sent ir el barrio como algo propio. } \\
\text { Sent irse apegado al barrio. } \\
\text { Intención de cont inuar viviendo en el } \\
\text { barrio. } \\
\text { Gisto por vivir en ese barrio. } \\
\text { Sent irse del bario. }\end{array}$ \\
\hline IDENTFCAGáN & SMBáபCA & $\begin{array}{l}\text { Dferencia con đt ros barrios. } \\
\text { Memoria personal en lugares del barrio. } \\
\text { Orgul lo de pert enecer a ese barrio. } \\
\text { Nombre del barrio. } \\
\text { Definición del lugar de donde uno dce } \\
\text { que es cuando le preguntan de dónde es. }\end{array}$ \\
\hline & Cotidanas & $\begin{array}{l}\text { Acciones real izackas en el barrio. } \\
\text { Compra en el barrio, pasar tiempo en el } \\
\text { barrio, relación, conocimient oy confianza } \\
\text { con gent e del barrio, ubicación de la red } \\
\text { de relaciones primarias y secunclarias. }\end{array}$ \\
\hline AOAONES & $\begin{array}{l}\text { Relacionadas confut uros } \\
\text { proyectos de barrio }\end{array}$ & $\begin{array}{l}\text { Acciones en relación al barrio. } \\
\text { Pertenencia a ent icades del barrio, } \\
\text { informarse delo que pasa en el barrio, } \\
\text { conocimiento de activicades organizadas } \\
\text { en el barrio, asi stencia a las actividades, } \\
\text { asistencia a act ivicades colect ivas, } \\
\text { asistencia a las fiestas del barrio } \\
\text { compromiso y visión hacia el barrio. } \\
\text { conocimient o de los proyectos, la opinión } \\
\text { propia es tenida en cuenta, iniciat iva de } \\
\text { los responsables compartiendo las } \\
\text { decisiones, iniciativa de la gente del } \\
\text { barriosin compart ir decisiones, ini ciat iva } \\
\text { de la gente del barrio compartiendo } \\
\text { decisiones desde el principio. }\end{array}$ \\
\hline
\end{tabular}

Fuente. Elaboración propia con base en a Vidal y Pol, 2005.

Dentro del modelo de análisis de la apropiación, la dimensión de la acción se divide en tres componentes que corresponden con las acciones cotidianas en el lugar, las acciones orientadas hacia el lugar, y acciones en torno a los proyectos de futuro del lugar. Esta división en tres componentes permite precisar la dimensión de la acción, en la que se señalan principalmente las interacciones sociales cotidianas, las prácticas, las actividades más habituales y las menos habituales, e incluso las de carácter ritual. Los indicadores que configuran la dimensión de la identificación, por su parte, recogen tres procesos implicados en la identificación mencionados por Graumann (1983): identificar el entorno, ser identificado por el entorno e identificarse con el entorno. En cuanto a la dimensión que contempla el apego al lugar, se encuentra el hecho de sentirse del barrio, querer seguir viviendo en el barrio, y todo ello marcado por una componente simbólica y afectiva. 


\section{D) Resultados y discusión}

\section{Primera dimensión de análisis: apego al lugar (componente simbólica)}

La primera dimensión de análisis de la apropiación hace referencia a los lazos afectivos que se tienen sobre un espacio o territorio dado. Son los sentimientos de carácter positivo, que se determinan basándose en las preferencias y deseos de los habitantes, ya que el apego implica un sentimiento de seguridad. Este sentimiento se manifestó durante las entrevistas realizadas (en el marco de la tesis doctoral)al interior del caso de estudio, donde uno de los referentes expresó: "El barrio es como una casa, lo querés hacer y queremos que cada día esté más lindo y dejar el ejemplo para nuestros hijos de que con unidad todo se puede, no hay un no posible, todo es posible" (Rodriguez Tarducci, 2020). La mayoría (60\%) de las personas censadas en el año 2017, en el marco del proyecto de investigación y las mesas de trabajo permanentes (MTP), coincidió en que el barrio en cierta medida había mejorado. No obstante, un gran número de vecinos sostuvo que el barrio había empeorado o que se mantenía igual hacía años. En este sentido, el $28 \%$ indicó que, en parte, había mejorado en algunas cuestiones y en otras empeoró, el $18 \%$ respondió que el barrio siempre está igual, y el $11 \%$ respondió que solo había empeorado. Con base en estos resultados, cabe señalar que las mejoras que los vecinos censados parecen encontrar en el barrio, rápidamente son identificadas por ellos en relación con la iniciativa colectiva. Manifiestan que, si se produjeron cambios positivos en relación con la infraestructura y la calidad de vida, tuvieron que ver con la unión y la insistencia, y no únicamente con la acción del Estado municipal en materia de intervención. A raíz de esta pregunta surgieron otros interrogantes que merecen ser analizados en relación al posible anhelo de algunos vecinos de vivir en otro barrio. Las respuestas en torno a esta pregunta representan un indicador del apego al lugar que sienten los residentes dentro de la componente simbólica de la apropiación. En este sentido, los resultados arrojaron que a un 54\% de las personas censadas le gustaría permanecer en el barrio. Si bien la mayoría de los vecinos pretende continuar viviendo en Puente de Fierro, muchos de ellos manifiestan que para que su deseo fuera completo en un $100 \%$ sería necesaria la intervención municipal para brindar las condiciones mínimas de habitabilidad que necesitan. Cabe destacar que muchos de los encuestados pertenecen a segunda o tercera generación de familias en el barrio, lo cual incrementa el sentido de pertenencia en relación con el apego al lugar por la memoria familiar, infancia y experiencia de vida en el barrio.

\section{Segunda dimensión de análisis: identificación (componente simbólica)}

Esta categoría incluye aquellos procesos identitarios que tienen relación con la categorización personal en la que figuran las manifestaciones de identidad del lugar, identidad urbana e identidad social urbana (Pol y Valera, 1994). Es preciso mencionar que todas ellas se encuentran conformadas por recuerdos, preferencias personales, memorias o cualquier otra referencia originada en la vida de las personas que guarde alguna relación con los espacios habitados (Vygotsky, 1934, citado en Pol, 1994). Tanto el apego al lugar como la identificación tienen una componente simbólica que tiene que ver con el hecho de que los espacios "tienen la capacidad de aglutinar determinados significados en su seno, es decir, tienen la capacidad de cargarse de significado simbólico" (Pol y Valera, 1994). En este sentido, algunos eventos -como la gran inundación que sufrió el territorio del Partido de La Plata el 2 de abril del año 2013 y que fue causante de grandes estragos en el barrio-también ponen de manifiesto la identificación entre los actores y el territorio.

Después de la inundación (del 2 de abril de 2013) nos dimos cuenta que somos todos hermanos, pasamos por un momento muy feo y no nos importaba nada más que estar todos juntos. Nos dimos cuenta que unidos podemos lograr muchas cosas (Vecino de Puente de fierro, comunicación personal, 2018). 
El hecho de que los vecinos se identifiquen como parte de uno u otro lugar dentro de la totalidad que representa al barrio pone de manifiesto la componente identitaria de la apropiación que existe en Puente de Fierro. En este sentido, los grafitis o murales en las calles de Puente de Fierro son un ejemplo que representa algunas manifestaciones de las estrategias a través de las cuales los individuos o grupos establecen vínculos afectivos o identificativos con el entorno o territorio que habitan. Sirven como indicadores que en algunos casos refieren a memorias dentro del barrio, representados en personajes y situaciones vividas, que forman parte de la experiencia, no siempre negativas de habitar (Figura 4). Los vecinos manifestaron orgullo de pertenecer al barrio, pero aun así suelen marcar diferencias entre distintos sectores a través de expresiones tales como: "aquella zona es más peligrosa que esta" o "esta parte del barrio es diferente porque acá somos gente de trabajo". Sin embargo, los vecinos entrevistados y censados coinciden que el barrio responde a un mismo nombre, Puente de Fierro, que los identifica a todos simbólicamente, con excepción del barrio Evita, expansión del barrio original que es interpretada por los vecinos como una extensión, la más reciente, del asentamiento original y que lleva otro nombre.

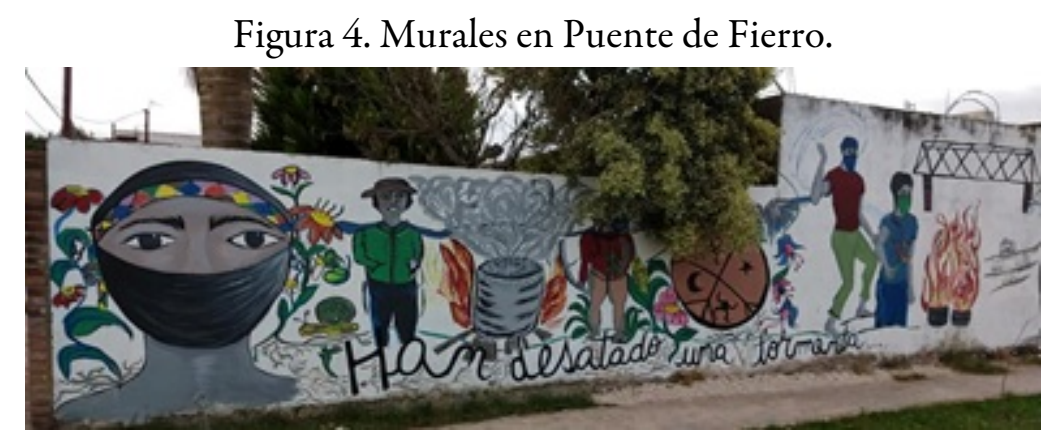

Fuente. Imagen tomada en Puente de Fierro entre 2016-2020 en el marco de las MTP.

Cuando se pregunta a algún habitante del barrio de dónde es y a dónde pertenece, la respuesta es "Puente de Fierro", indistintamente de la zona del interior a la que se haga referencia, o de dónde se encuentre ubicada su vivienda o del lugar donde haya nacido. Aun así, se marca la diferencia con otros barrios cercanos, es decir, se encuentran algunas identificaciones con lugares al interior del barrio, pero al comparar con otras urbanizaciones, la identificación y la representación en el imaginario colectivo de los vecinos del barrio es mayor. El sentido de pertenencia y la identificación con el barrio se manifiestan a través de las acciones que se realizan en el barrio, como se profundizará en el siguiente apartado, dado que las mismas se proponen para la totalidad del barrio, sin identificar de manera sectorizada uno u otro lugar. Por el contrario, los mismos vecinos mencionan que es necesario que todos formen parte de las noticias y decisiones que se toman en relación con el funcionamiento del barrio. Un ejemplo de ello lo constituyen las Mesas de Trabajo Permanente en el barrio (MTP), a las que acuden residentes de todos los lugares del interior de Puente de Fierro, y de todas las organizaciones sociales, ya que, en su mayoría, entienden que el acto de formar una unidad otorga beneficios mayores en cuanto a posibilidades de mejora.

\section{Tercera dimensión de análisis: las acciones}

Las acciones constituyen manifestaciones que pueden darse a nivel individual o de manera colectiva, cuando este proceso es reconocido y compartido por una comunidad o un amplio número de personas. Por ello, se busca detectar la carga de significados que la comunidad ha establecido en los espacios o prácticas, la cual es resultado de "una construcción social que opera entre las personas que configuran esta comunidad o que utilizan este espacio o se relacionan con/en él” (Valera, 1996). La participación y la comunicación son mecanismos que favorecen la apropiación; la primera permite coordinar acciones orientadas a la 
transformación, mientras que la segunda dota de contenido a la identificación del barrio. A través de la acción sobre el entorno, las personas y los grupos transforman el espacio, y dejan en él su impronta, es decir, señales y marcas cargadas simbólicamente. Mediante la acción, la persona o grupo incorpora el entorno en sus procesos cognitivos y afectivos de manera activa y actualizada. Las acciones dotan al espacio de significado individual y social, a través de los procesos de interacción (Pol, 1996). Cabe mencionar que, en términos metodológicos, las acciones hacia el futuro del barrio, junto con las acciones de carácter cotidiano (como hacer la compra en el barrio y relacionarse con los vecinos) y las acciones orientadas hacia el barrio (como asistir a las actividades que se realizan en el barrio) se operacionalizaron a través de diferentes indicadores, en cuestionarios y entrevistas, al igual que las demás dimensiones (identificación y apego al lugar). Lo anteriormente expresado se pone de manifiesto en las siguientes palabras de un vecino del barrio, al señalar que:

Nosotros queremos hacer de Puente un territorio posible trabajando en equipo; somos una gran familia, respetando el trabajo de cada institución, siempre tratando de solucionar cosas, con muchísimos proyectos para el barrio (Referente barrial, comunicación personal, 2019).

\section{Acciones cotidianas - Acciones realizadas en el barrio}

Entre las acciones cotidianas que se realizan en el barrio Puente de Fierro se encuentran actividades diarias en el barrio, tales como pasar tiempo en él, la relación, conocimiento y confianza con gente del barrio y la ubicación de la red de relaciones sociales primarias y secundarias entre vecinos (Figura 5). Si bien estas acciones tienen un carácter cotidiano, algunas de ellas, que merece la pena mencionar, acontecen durante los fines de semana, como las ferias de ropa y las ollas comunales, en las que la comunidad participa. Se ha mencionado que la participación y la comunicación son mecanismos que favorecen la apropiación, y esto se manifiesta en el fortalecimiento de las redes sociales a partir de las acciones cotidianas realizadas en el barrio.

Figura 5. Acciones cotidianas en Puente de Fierro.

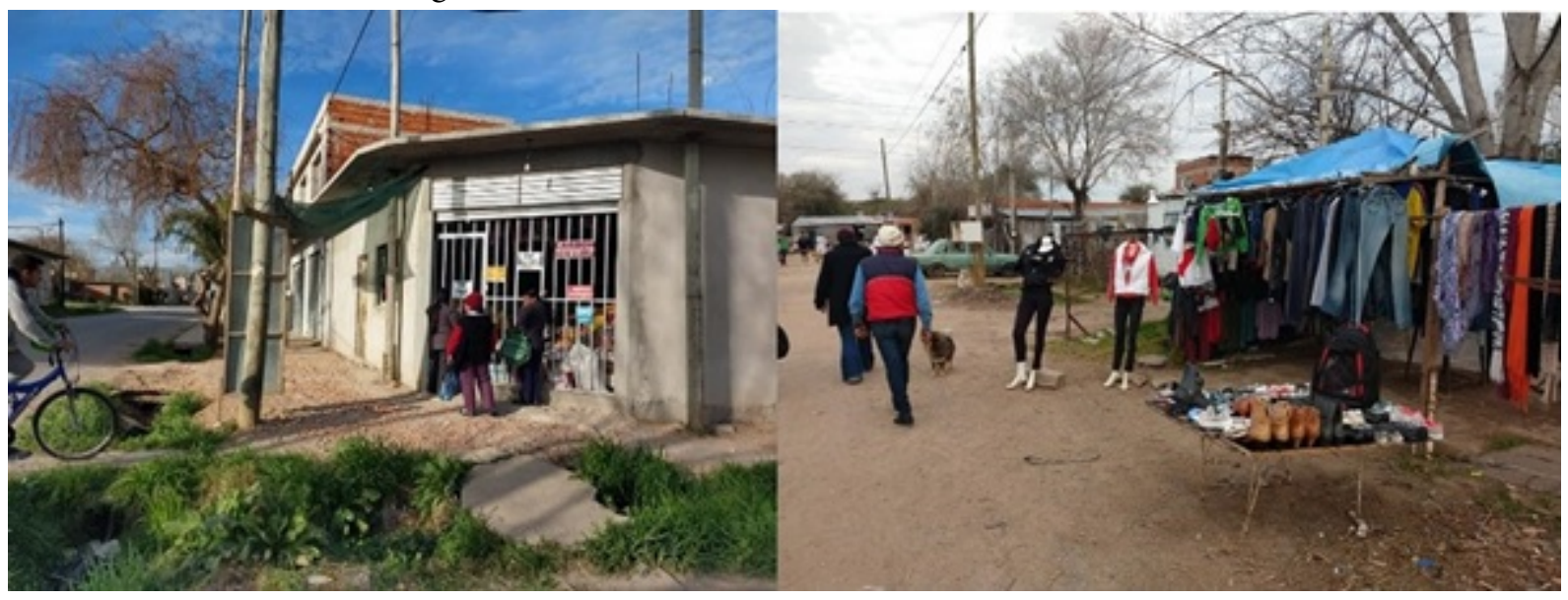

Fuente. Imagen tomada en Puente de Fierro entre 2016-2020 en el marco de las MTP.

Otras acciones cotidianas que se realizan en el barrio tienen que ver con la actividad de los comedores, merenderos y copas de leche, que diariamente alimentan a los niños del barrio que llegan después del colegio para tomar la que tal vez sea su única comida de la jornada. En la figura 6 se observan dos de los comedores a los cuales se ha podido acceder a través de sus organizadoras, mujeres referentes del barrio que trabajan colectivamente todos los días para asegurar el alimento de los niños a través de donaciones principalmente. 
Figura 6. Comedores y merenderos en Puente de Fierro.

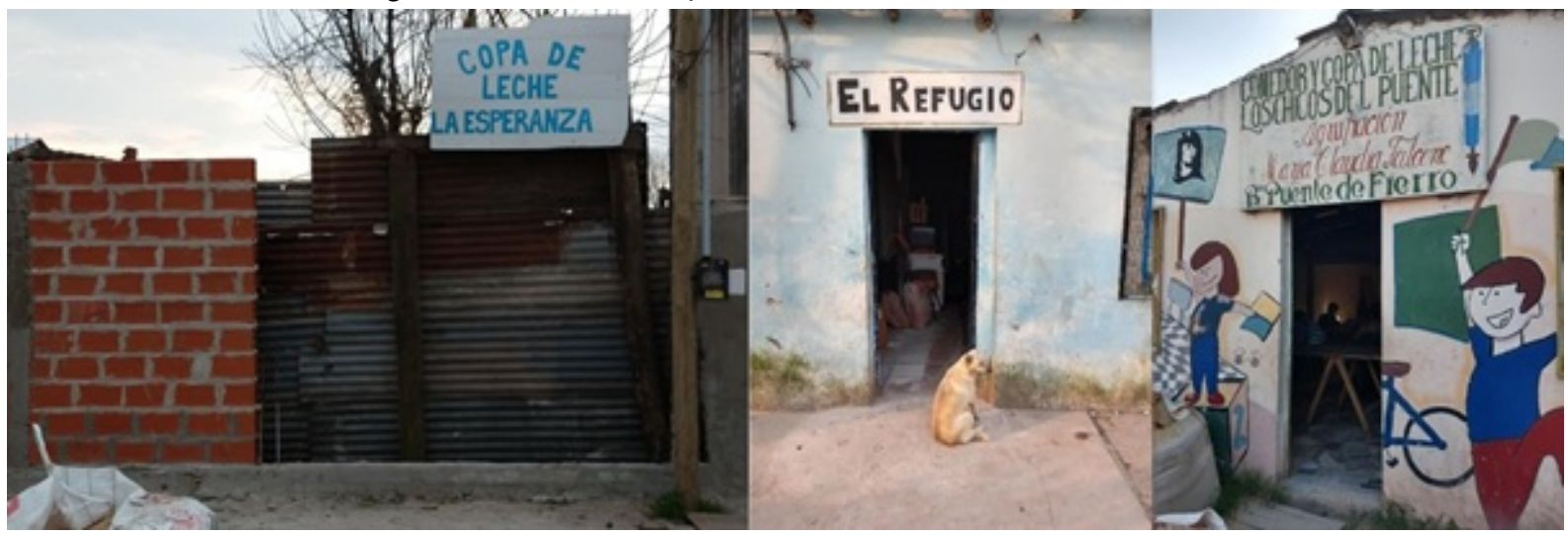

Fuente. Imagen tomada en Puente de Fierro entre 2016-2020 en el marco de las MTP.

Todas estas acciones se realizan a diario en Puente de Fierro, y manifiestan la fuerte apropiación que hacen los vecinos del lugar; lo mismo ocurre con otras acciones vinculadas a distintas actividades como las deportivas y las de recreación, como se observa en la figura 7. Los niños y jóvenes del barrio suelen utilizar los espacios como la "placita del barrio" y "la canchita" como espacios de reunión para jugar al fútbol, uno de los deportes preferidos, del mismo modo se desarrollan otro tipo de actividades de recreación como las murgas o reuniones de la banda de rap de Puente de Fierro.

Figura 7. Espacio público, recreativo y deportivo en Puente de Fierro.

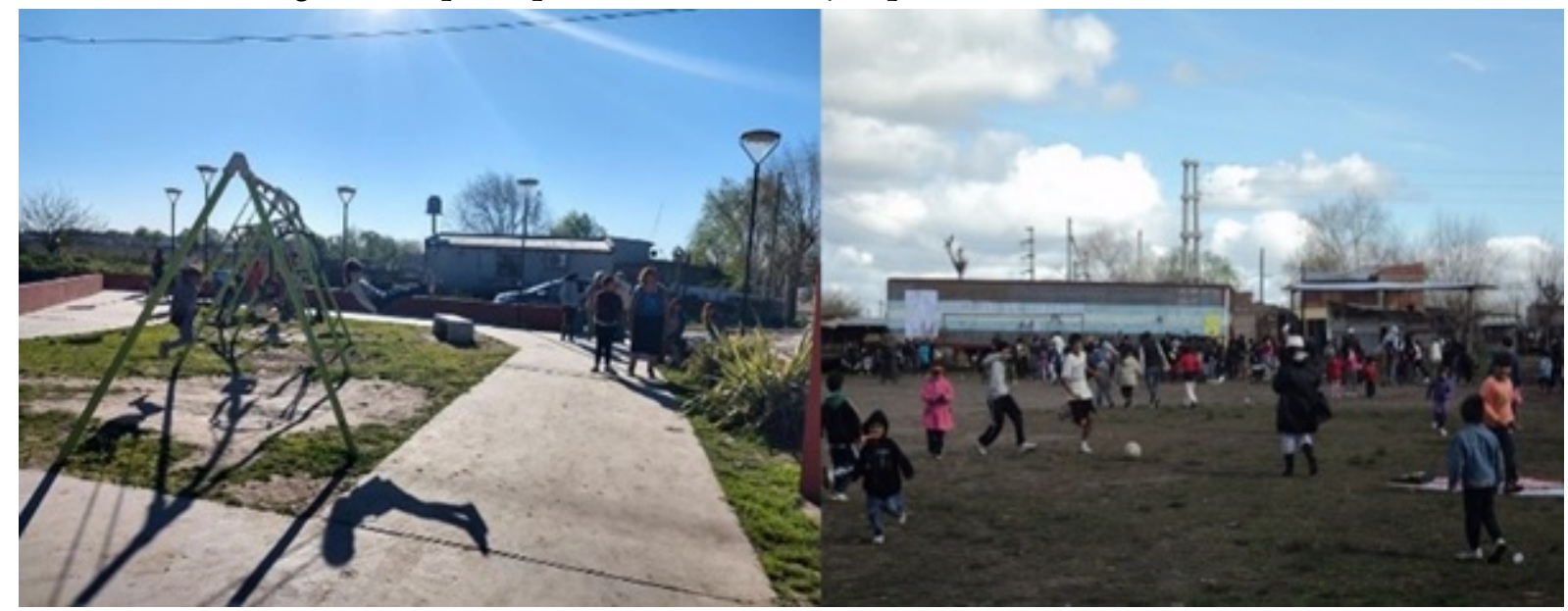

Fuente. Imagen tomada en Puente de Fierro entre 2016-2020 en el marco de las MTP.

\section{Acciones orientadas hacia el lugar - Acciones en relación al barrio}

Las acciones al interior de una comunidad pueden articularse en torno a su carácter colectivo y compartido, y se encuentran muy vinculadas con la participación de los vecinos en relación con las acciones hacia el barrio. Estas acciones tienen que ver con la pertenencia a entidades del barrio, la información sobre lo que pasa en el barrio, el conocimiento de actividades organizadas en el barrio, la asistencia a actividades colectivas, como, por ejemplo, las fiestas del barrio. Un ejemplo de ello tiene que ver con las cuestiones relacionadas a las condiciones ambientales y de saneamiento. Como se observa en la figura 9 , acciones como la de recoger la basura y limpiar las calles ponen de manifiesto el conocimiento y el interés de los vecinos de participar de actividades de mejora del barrio. En la imagen se observa cómo los vecinos, luego de recibir donaciones de barriles por parte de la empresa YPF, decidieron trabajar en conjunto y pusieron en marcha 
un proyecto comunal para transformarlos en cestos de basura que colocaron en distintas esquinas de todo Puente de Fierro. Esta acción pone de manifiesto la intención de enseñar a los demás vecinos del barrio a colocar los residuos en cestos y a cuidar el entorno. Si bien muchas de estas acciones deberían proceder por parte del Estado (como por ejemplo la colocación de cestos), cuando esto no sucede, son los mismos vecinos, organizaciones sociales y cooperativas, los que buscan alternativas y soluciones.

Figura 8. Acciones Orientadas hacia el lugar en Puente de Fierro.

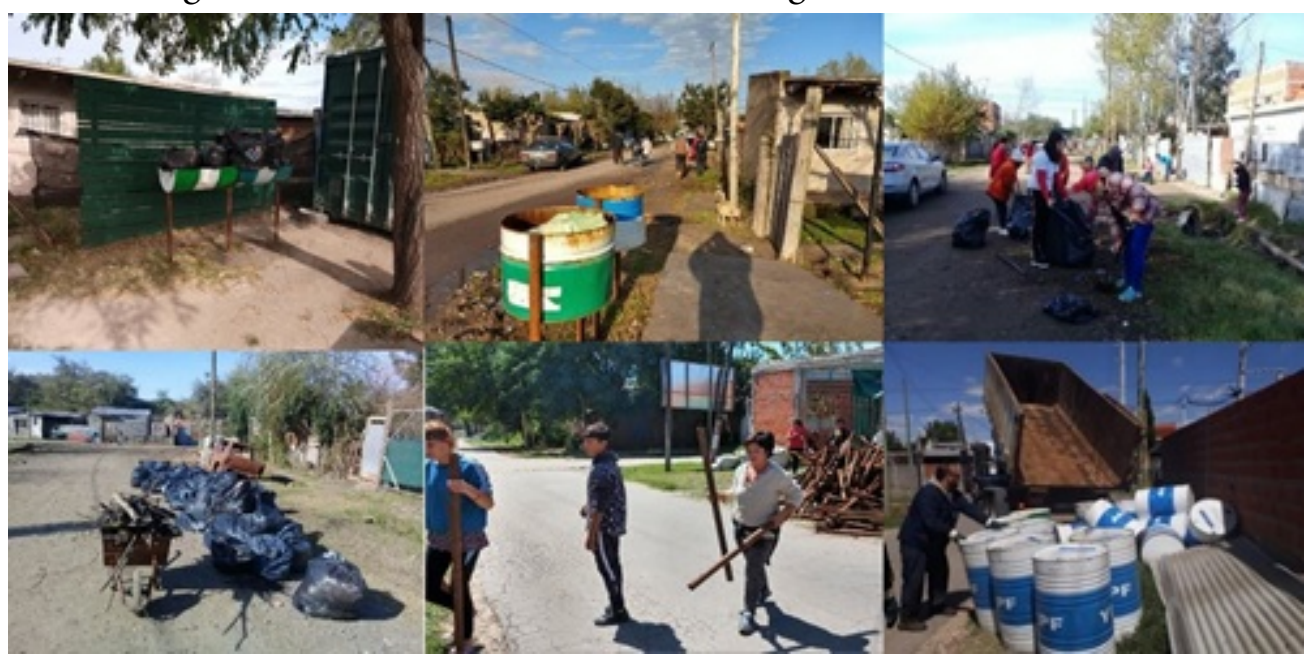

Fuente. Imagen tomada en Puente de Fierro entre 2016-2020 en el marco de las MTP.

Entre las acciones que se realizan en relación al barrio, también pueden destacarse aquellas que forman parte de proyectos en conjunto con la Universidad Nacional de La Plata (proyectos de extensión) y diversas ONG, cuya finalidad consiste en ofrecer algún tipo de servicio o conocimiento sobre una temática específica. A modo de ejemplo, en la figura 9 se observa parte de las jornadas de vacunación para perros en las calles del barrio Puente de Fierro. Esta acción se llevó adelante pensando en el futuro del barrio, no solo en relación con la salud de los animales, que en su mayoría están en las calles y comen de la basura, sino fundamentalmente en beneficio de las condiciones de habitabilidad y salud de los residentes del barrio. Otro proyecto de los de mayor relevancia en relación con las acciones orientadas hacia el lugar lo constituye la iniciativa de ladrillos ecológicos para la construcción de viviendas a partir de la reutilización de desechos plásticos. Esta iniciativa no solo la llevan adelante los mismos vecinos en comedores y copas de leche en el barrio, sino que, además, las organizaciones sociales también reciben donaciones de los mismos. A partir de la iniciativa de los ladrillos ecológicos, los vecinos buscan la construcción de viviendas sustentables y a bajo costo, lo que pone de manifiesto el sentido de pertenencia y la participación colectiva. 
Figura 9. Vacunación de animales, ladrillos ecológicos y participación colectiva en Puente de Fierro

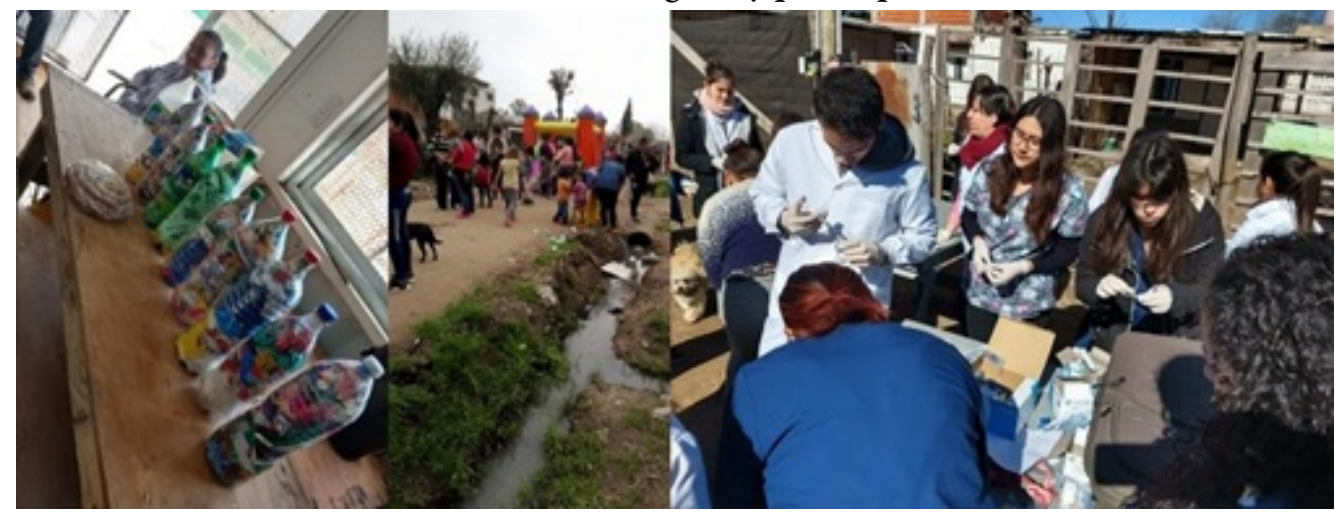

Fuente. Imagen tomada en Puente de Fierro entre 2016-2020 en el marco de las MTP.

Las acciones mencionadas evidencian un interés y una preocupación de parte de los vecinos por el presente y futuro del barrio, y hacen visible una fuerte apropiación del territorio. Del mismo modo se manifiesta el apego al lugar y la identificación simbólica que los residentes sienten en torno al barrio. El apego y la identificación son parte de la componente simbólica de la apropiación; en este sentido, ambas componentes (material y simbólica) no son excluyentes, sino que, por el contrario, se operacionalizan para una mejor interpretación del análisis, pero no surgen de manera aislada, sino que se manifiestan en conjunto, o dialécticamente.

\section{Acciones relacionadas con futuros proyectos del barrio - Compromiso y visión hacia el barrio}

Las últimas acciones que se consideran en el análisis de la apropiación son aquellas que se vinculan con los proyectos futuros del barrio; aquí se toman el compromiso y la visión como indicadores. Para el análisis de estas acciones se tiene en cuenta el conocimiento acerca de los proyectos futuros para el barrio y la opinión propia de los vecinos sobre los mismos, así como la iniciativa del barrio de compartir las decisiones. En este sentido, se mencionó anteriormente que desde el año 2016 se llevan a cabo mensualmente las Mesas de Trabajo Permanente (MTP), abiertas a los vecinos y a la comunidad en general, a la Universidad Nacional de La Plata y a otras universidades, a empresas, a actores de la gestión, etc. Las mismas tienen lugar en el barrio Puente de Fierro y surgieron por iniciativa de los vecinos, las organizaciones sociales y tienen participación activa de la Universidad de La Plata (figura 10).

Figura 10. Algunas de las mesas de trabajo permanente (MTP) en Puente de Fierro entre los años 2016 y 2020.

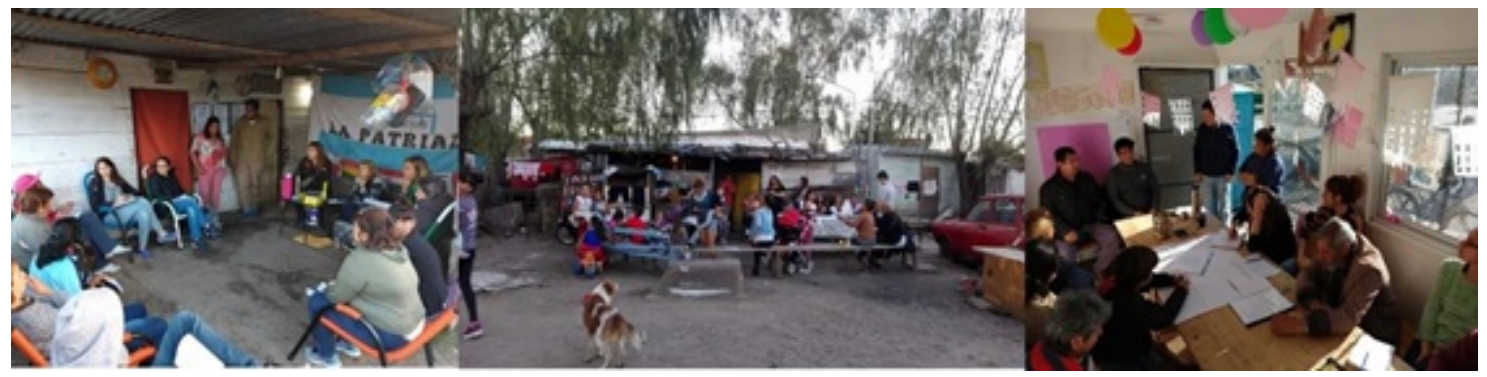

Fuente. Imagen tomada en Puente de Fierro entre 2016-2020 en el marco de las MTP. 
Durante las primeras MTP se plantearon temas que les interesaba tratar a miembros de organizaciones sociales y vecinos del barrio. La premisa para iniciar el diálogo entre actores consistió en cuatro preguntas guía: ¿quiénes somos?, ¿dónde estamos?, ¿qué queremos hacer?, y ¿qué estamos haciendo? Estas preguntas y las ideas que surgieron en más MTP desde el primer momento evidenciaron la acción transformadora de los vecinos y el compromiso de mejorar la situación del barrio. Como producto de la participación y constancia en la realización de las MTP surgieron varios proyectos en relación con el barrio, que dan cuenta del compromiso y la visión de los vecinos. Entre ellos se destaca la conformación de la revista del barrio Trazando Puentes (figura 11). En ella, los habitantes del barrio, junto con profesionales y tesistas de la Facultad de Comunicación Social (UNLP), encontraron una forma de comunicar y visibilizar las problemáticas en el barrio, y de este modo presentárselo a quienes no lo conocían.

Figura 11. La revista de Puente de Fierro (izq.) y el trabajo colectivo en las MTP (der.).

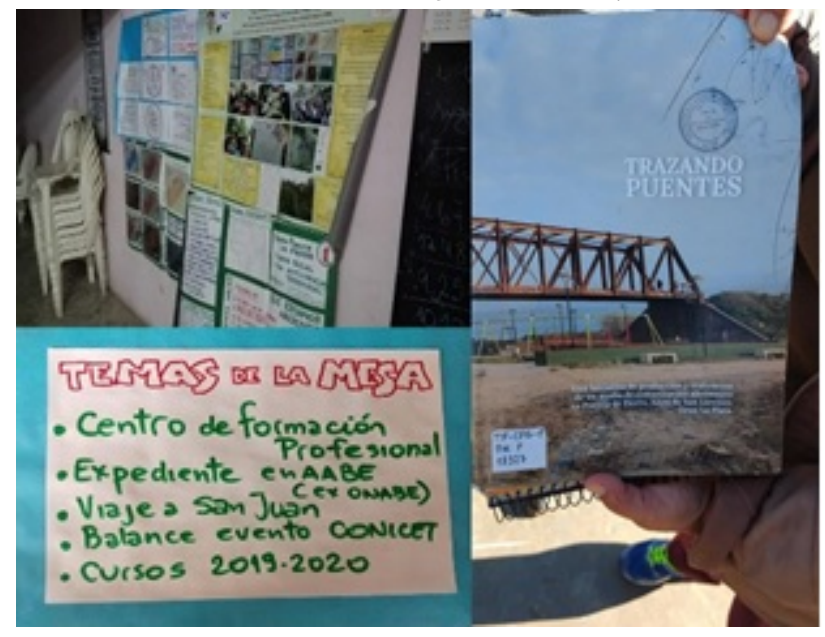

Fuente. Imagen tomada en Puente de Fierro entre 2016-2020 en el marco de las MTP.

Las acciones vinculadas con los proyectos del barrio, que ponen de manifiesto la apropiación territorial a partir del compromiso y visión, se evidencian también en el modo en que los vecinos se expresan en relación con los anhelos y el orgullo que sienten por las transformaciones que reconocen en el barrio, producto de la unión que generan espacios como las MTP.

En el día a día trabajamos en conjunto; se habla y se trabaja; es la unión del barrio. Costó un montón, pero la cuestión es que tiramos todos para que el barrio progrese, ahora se ven hechos fundamentales. Si nos retrotraemos al Puente de la época militar, te puedo decir que he vivido desde chica muchas cosas en el barrio, pero lo que se ha logrado ahora... los chicos tienen su plaza, es otra cosa (Fragmento de entrevista con referente barrial, disponible en Rodríguez Tarducci, 2020).

\section{Conclusiones}

La relación entre el modo colectivo que tienen los habitantes de Puente de Fierro de abordar los problemas del barrio, la acción hacia el barrio y la identificación con este invitan a continuar indagando en la relación entre los actores, el territorio, la construcción de lugares y la participación ciudadana. Queda demostrado que los objetos no son ajenos ni independientes del accionar de los actores; estos tampoco pueden ser abordados ni aprehendidos directamente en sí mismos, sino a través de las prácticas, ya que solo se construyen y reconstruyen en las acciones a la luz de lo que se hace. Es a partir del resultado de las acciones que se pone de manifiesto en la empiria la teoría de Santos (1996b), que asegura que los objetos son el testimonio de la acción, al mismo tiempo que ponen en evidencia la transformación del territorio que los actores llevan adelante a través de sus acciones dentro del proceso de ocupación y apropiación territorial. 
Del análisis se desprende el valor y significado de las interacciones simbólicas que se producen entre los actores sociales que habitan un territorio, y es precisamente a través de sus acciones que lo transforman en lugar. A partir del establecimiento de dimensiones, componentes e indicadores, se generaron herramientas que permitieron operacionalizar el concepto de apropiación. Del mismo modo, a partir de las entrevistas y la participación activa en el barrio se pudo profundizar en la función, el valor y significado que los habitantes de Puente de Fierro construyen a partir de sus percepciones y experiencias. De este modo fue posible comprender que los procesos de interacción con el territorio confirman la relación que se origina entre las personas y los lugares. En tal sentido, resulta posible afirmar las acciones colectivas ponen de manifiesto y a la vez fortalecen el sentido de pertenencia, el apego y la identificación con el lugar.

\section{REFERENCIAS}

Bourdieu, P. (2000) La miseria del mundo. Bs As: Fondo de Cultura Económica.

Graumann, C.F. (1983). On múltiple identities. International Social Sciences Journal, 35, 309-321.

Gumuchian, H. (1991). Representations et aménagement du territoire. París: Económica.

ONU - HABITAT. Por un mejor futuro urbano. Recuperado de: https://onuhabitat.org.mx/index.php/hacer-de-lo s-asentamientos-informales-parte-de-la-ciudad.

Pol, E. (1996). La apropiación del espacio. En L. Î́niguez y E. Pol (Eds.), Cognición, representación y apropiación del espacio. Collecció Monografies Psico-Socio-Ambientals (vol.9, pp. 45-62). Barcelona: Publicacions de la Universitat de Barcelona.

Pol, E. (2002). El modelo dual de la apropiación del espacio. En Psicología y Medio Ambiente. Aspectos psicosociales, educativos y metodológicos (pp. 123-132). España: Unidad de Investigación Persona-Ambiente, Universidad de A Coruña.

Reguillo, R. (2005). La construcción simbólica de la ciudad. Ciudad, desastre y comunicación. México. ITESO.

Rodríguez Tarducci, R. (2020). Informalidad urbana en el partido de La Plata: Análisis del proceso de ocupación y apropiación territorial, 1989-actualidad. (Tesis de doctorado en geografía inédita). Universidad Nacional de La Plata, Facultad de Humanidades y Ciencias de la Educación, Buenos Aires, Argentina.

Santos, M. (1996a). A natureza do espaço. Técnica e tempo. Razão e emoção. São Paulo: Hucitec.

Santos, M. (1996b). De la totalidad al lugar. Barcelona: Oikos-Tau.

Schneider, S. y Peyre (2006). Territorio y enfoque territorial: De las referencias cognitivas a los aportes aplicados al análisis de los procesos sociales rurales. En M. Manzanal; G. Neiman y M. Lattuada (Eds.). Desarrollo Rural. Organizaciones, Instituciones y Territorio (pp. 71-102). Buenos Aires: Ciccus.

Valera, S., y Pol, E. (1994). El concepto de identidad social urbana: una aproximación entre la Psicología Social y la Psicología Ambiental. Anuario de Psicología, 62, 5-24.

Valera, S. (1996). Análisis de los aspectos simbólicos del espacio urbano. Perspectivas desde la psicología ambiental. Revista de Psicologia. Universitas Tarraconensis, 18, 63-84.

Vidal, M., Tomeu, P., y Urrútia, E. (2005). La apropiación del espacio: una propuesta teórica para comprender la vinculación entre las personas y los lugares. Anuario de Psicología, 36(3), 281-98. Recuperado de https://www. redalyc.org/comocitar.oa?id=97017406003.

\section{Notas}

1 El presente artículo forma parte de la tesis de doctorado denominada "Informalidad urbana en el Partido de La Plata: Análisis del proceso de ocupación y apropiación territorial 1989-actualidad” en el marco del Doctorado en Geografía de la Facultad de Humanidades y Ciencias de la Educación, Universidad Nacional de La Plata, Argentina (Rodríguez Tarducci, 2020). 
2 Proyecto PIO UNLP CONICET 2014-2016 es "Estrategias para la Gestión Integral del Territorio. Vulnerabilidades y Procesos de Intervención y Transformación con Inteligencia Territorial. Métodos y técnicas científicas ambientales, sociales y espaciales: Dos casos en el Gran La Plata”. Con participación de más de 50 investigadores, profesores y tesistas de la UNLP, CONICET y CIC de las Ciencias Exactas, Sociales y Naturales.

3 Se trata de una iniciativa innovadora de gestión integral del territorio en la urbanización informal más grande de La Plata, Buenos Aires, Argentina, que mediante la ejecución de más de treinta mesas mensuales de trabajo ha producido notables resultados a través del acompañamiento ala construcción de políticas públicas. Proyecto de Investigación PIO UNLPCONICET 2104-2016. Actualmente Proyecto de Investigación "La Ciencia y la Gente, otras Políticas Públicas" con sede en IdIHCS UNLP-CONICET. http://idihcs.fahce.unlp.edu.ar/territoriosposibles/puente-de-fierro-territorio-p osible-mesa-de-trabajo-permanente-y-agenda-cientifica-2014-2026-pio-om/ 